\title{
Exploring Academic Library Users' Preferences of Delivery Methods for Library Instruction
}

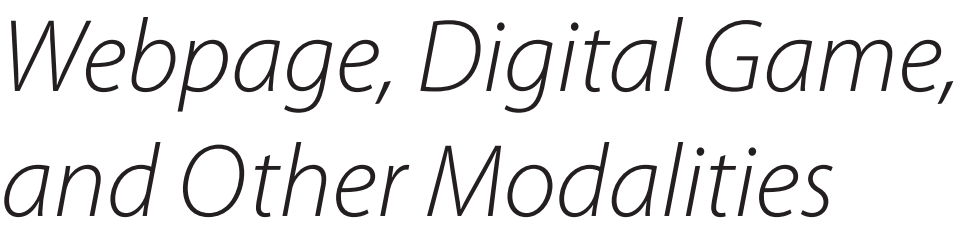

This article examines research on academic library user preferences related to five communication media used to facilitate two forms of library instruction. The corresponding study began with a survey instrument administered to forty-two participants over a three-week period during the fall of 2006. The authors subsequently applied three nonparametric scaling methods to the data set. Data analysis indicates an overall preference for the $2 D$ webpage approach, as well as notable enthusiasm for the 3D immersive graphical user interface, the principal user interface throughout current digital gaming technologies. An overall lack of preference for the audio-only communication medium is also evident in the results.

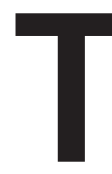
his article expands upon recent library and information science (LIS) discourse related to digital gaming by investigating user perceptions of digital games (i.e., video, computer, and online games) relative to other communication media currently used by academic libraries to facilitate library instruction. ${ }^{1}$ Adapting previous definitions for the purposes of this discussion, library instruction describes the collective educational efforts-formal and informal_of an academic library. ${ }^{2}$ The primary question driving the present research is, Do aca- demic library users prefer digital game systems over other comparable communication media (i.e., information presentation formats or delivery approaches) for engaging library instruction?

Current digital gaming technologies, such as Blizzard Entertainment's massively multiplayer online role playing game (MMORPG) World of Warcraft, provide users with functionality far beyond the traditional competitive aspects of games. They also exemplify social computing. Because of three primary factors, modern digital games are significantly different from their predecessors. These three factors are (1) the exponential increase in computing power, leading to (2) the development of more realistic gaming experiences (i.e., three-dimensional direct manipulation user interface), and (3) the Internet. Increasingly, digital game users expect both immersive and collaborative systems in which meaningful game play experiences include user-to-user communication through text, voice, and even virtual body movement. Ultimately, modern digital games are not just competitive environments; through the Internet they fundamentally act as communication media. Interdisciplinary literature on various sociocognitive aspects of digital-game use reinforces this assertion. ${ }^{3}$

\section{Michael J. Robertson and James G. Jones}

Michael J. Robertson is a doctoral candidate enrolled in the College of Information, Library Science, and Technologies at the University of North Texas in Denton. James G. Jones is Assistant Professor, Learning Technologies Department, College of Information, Library Science, and Technologies, University of North Texas. Submitted for review August 23, 2007; revised and accepted for publication November 19, 2007.

Reference \& User Services Quarterly, vol. 48 , no. 3, pp. 259-269

(c) 2009 American Library Association. All rights reserved.

Permission granted to reproduce for nonprofit, educational use. 
This article begins by discussing two components of library instruction-providing users with spatial (directional) information on physical library layout and educating users on information literacy topics—and considers how the application of a 3D immersive graphical user interface (GUI), the principal user interface adopted by most current digital games, may benefit library users. Next, it reviews a survey in which forty-two participants ranked according to preference five communication media, including a 3D immersive GUI, relative to the aforementioned two types of library instruction. Remaining sections summarize research findings derived using three nonparametric scaling methods, examine select interpretations of those findings, and discuss their potential contribution to future research.

\section{BACKGROUND}

Outside the LIS community, a prevalent topic of discussion related to digital gaming research is the 3D immersive GUI. ${ }^{4}$ For example, some researchers suggest that, in comparison to more traditional communication media, the 3D immersive GUI provides users with an increased real-world sense of spatial context (i.e., spatial information enabling a user to orient and navigate the related environment). Moreover, some researchers suggest that spatial cognition is an important contributor to meaningful learning experiences but is less so in the areas of traditional information access and retrieval. ${ }^{5}$

Many of the current theoretical approaches to cognition, such as trajectories of participation and communities of practice, view learning as a primary activity of human information processing; these approaches reflect to varying degrees historically significant literature by Dewey, Kelly, Bruner, and Vygotsky. ${ }^{6}$ Additionally, research suggests that digital animation may support users' abilities to simplify structure during learning activities. ${ }^{7}$ This assertion is congruent with research in data-mining presentation techniques and runs parallel with findings in cognitive neuroscience that indicate increased dopamine levels in digital game users during user-system interaction periods. ${ }^{8}$

In practice, academic libraries do far more than provide access to information; they also act as both formal and informal educational agencies. ${ }^{9}$ Academic libraries educate users through a range of communication media, stretching from simple pamphlets to extensive instructional websites. The following two subsections expand upon the two forms of library instruction previously mentioned: providing users with spatial information on physical library layout and educating users on information literacy topics. These two forms of library instruction-informal and formal, nontraditional and traditional-provide the basis for the two main questions of the survey instrument discussed in the study section.

\section{Library Layout}

Walking into a physical library for the first time and attempting to locate a particular area within the facility can potentially be a frustrating experience. Although not prototypical to many LIS researchers, such a scenario exemplifies a common information-seeking experience that occurs daily in academic libraries. Keefer suggests that academic library users under time constraints and other stresses are more likely to have difficulty conducting independent research; often these users fail to see directional signs and other communication media providing spatial information on the facility. ${ }^{10}$ Voelker reinforces this aspect of library instruction in her discussion of freshman users, information literacy instruction, and library anxiety. ${ }^{11}$ Ultimately, acquiring spatial information about a physical library facility is necessary for all of the institution's users.

Consumer research indicates that information presentation formats significantly affect information acquisition and subsequent learning processes in users. ${ }^{12}$ Thus, when attempting to convey spatial information, choice of communication media can influence the quality of the user's learning experience. Additionally, because of human beings' primary dependence on visual perception, communication media can vary greatly, not only in the literal information provided, but also in the amount of spatial context they offer the user. For academic libraries, frequent means of communicating spatial information include pamphlets, strategically placed signs, and online guides through an institution's Web presence.

From a sociocognitive perspective, proponents of the 3D immersive GUI suggest that the visual navigation features of this design approach, coupled with connection to a library's Web presence, provides a potent interface for the more visually inclined users. Jones and Bronack refer to this ability as cognitive scaffolding. ${ }^{13}$ Consider that, in physical reality, users employ various voluntary and involuntary body movements to communicate, that is, facial expressions, hand signals, posture, and so on. Digital games allow users to process information through audio dialogue, text, and avatar (i.e., virtual-self) movement. The 3D immersive GUI approach also permits users to en- 
gage one another within an entirely neutral digital reality, an important benefit for freshmen sensitive to their own abilities to integrate into the physical library environment. ${ }^{14}$

\section{Information Literacy}

For most academic libraries, a primary component of their service missions is to educate users on information literacy concepts and skills. Such instruction often occurs either in a face-to-face workshop setting or online and may include educating users in evaluating information resources, searching electronic bibliographic databases, and using other services offered by the institution such as interlibrary loan. Ultimately, the goal of information literacy instruction is to encourage library users to be independent researchers confident in their abilities to locate and use valid information both in physical and digital formats. ${ }^{15}$

While limited, digital games and information literacy instruction share some history. The first mention of any relationship is in 1982, with the advent of Citation, a digital game designed to teach young people basic information literacy skills. ${ }^{16}$ Currently there are a variety of approaches to using digital games in this context, ranging from providing online educational games that incorporate information literacy concepts to presenting an entire online digital library and its services through a 3D immersive GUI. ${ }^{17}$ In relation, Lewis describes using digital games to promote library services and reviews two UK programs wherein digital gaming technologies augment library instruction. ${ }^{18}$

Voelker stresses that academic libraries must reach beyond their physical facilities to meet the expectations of freshman users, while Doshi suggests that many academic library users view the physical facility and its services and staff as essentially boring. ${ }^{19}$ Research on Millennial users (individuals born after 1980) may shed some light on why freshman students and other Millennials perceive libraries and their constituent parts with little enthusiasm. Abram and Luther contend that Millennials differ considerably from previous user groups in nine fundamental ways, including multitasking as a fundamental pattern of behavior and game-like experiential learning strategies. ${ }^{20}$ Consider that Millennials are accustomed to quickly flashing digital interfaces as well as multitasking through numerous digital devices simultaneously. ${ }^{21}$ Parallel research suggests that, because of their interactive, participatory nature, digital games provide users with a wide range of multitasking opportunities. ${ }^{22}$ Additionally, Rise proposes that Millennials present an entirely new set of values and expectations driven by their overall affinity for digital technology, while Dede suggests that Millennials maintain a unique learning style grounded in ubiquitous, mediated immersion of digital content. ${ }^{23}$ Dede also proposes that Millennials are profoundly affecting traditional higher education, sparking institutional changes on strategic investments in physical plants, technology infrastructure, and professional development.

\section{Study}

Perception is the operationalized group of nested cognitive processes (e.g., attention, consciousness, and memory) from which users make sense of their external worlds. ${ }^{24}$ Understanding how users perceive the applicability of particular technologies in task-oriented contexts is extremely important to both LIS researchers and practitioners. The development of such knowledge aids decision makers in preparing a more accurate view of user expectations. Various interdisciplinary literature on technology adoption and use reinforces this assertion. ${ }^{25}$ In such research, user perceptions (i.e., psychological determinants such as perceived ease of use, perceived usefulness, and perceived user resources) are paramount. Ultimately, psychological determinants structure user attitudes toward adoption of a particular technology.

This research uses methods rooted in psychophysics, the measuring of users' perceptions of physical properties of environmental stimuli. ${ }^{26}$ Perceptual data contribute to research on the similarity and dissimilarity of stimuli as well as the estimation of perceptual magnitude between stimuli. ${ }^{27}$ Today, individuals in both academicand practice-based contexts use such methods to simplify data sets into underlying psychological constructs representing participants' perceptions of physical objects and alternative representations of physical objects. As such, the focus of this research is to measure the incoming perceptions of academic library users toward the five communication media, not familiarity or comfort with the media. Eisenberg, Oyarce, Rorissa, and Rorvig also use psychophysical methods in LIS research. ${ }^{28}$

During the fall of 2006, academic library users at the University of North Texas (UNT) were asked to participate in this study. Solicitation of participants occurred both by face-to-face contact and through e-mail. As shown in table 1, 43 percent of the participants reported being between nineteen and thirty years old ( $n=18)$. By comparison, the mean age of all students at the university during the study period was 24.4 years old. ${ }^{29}$ Participation involved users completing a short, paper-based 
survey. In total, forty-two users participated, a sufficient number to ensure that the communication media were significantly different. ${ }^{30}$ The five communication media (i.e., scalable objects and psychological stimuli) are as follows:

- Paper-based pamphlet (object 1), shown in figure 1

- 2D webpage (object 2), shown in figure 2

- 3D immersive GUI (object 3), shown in figure 3

- Actual survey included a graphic representation (objects 4 and 5)

\section{INSTRUMENT}

The survey instrument follows a generally accepted format for acquiring data through responses to pairwise (paired) comparisons, a research method used in communication studies, zoology, public health, and various other disciplines. In short, the pairwise comparison method requires a participant to vote on objects presented in pairs relative to a given question or scenario. By counting the
Figure 1. Paper-Based Pamphlet

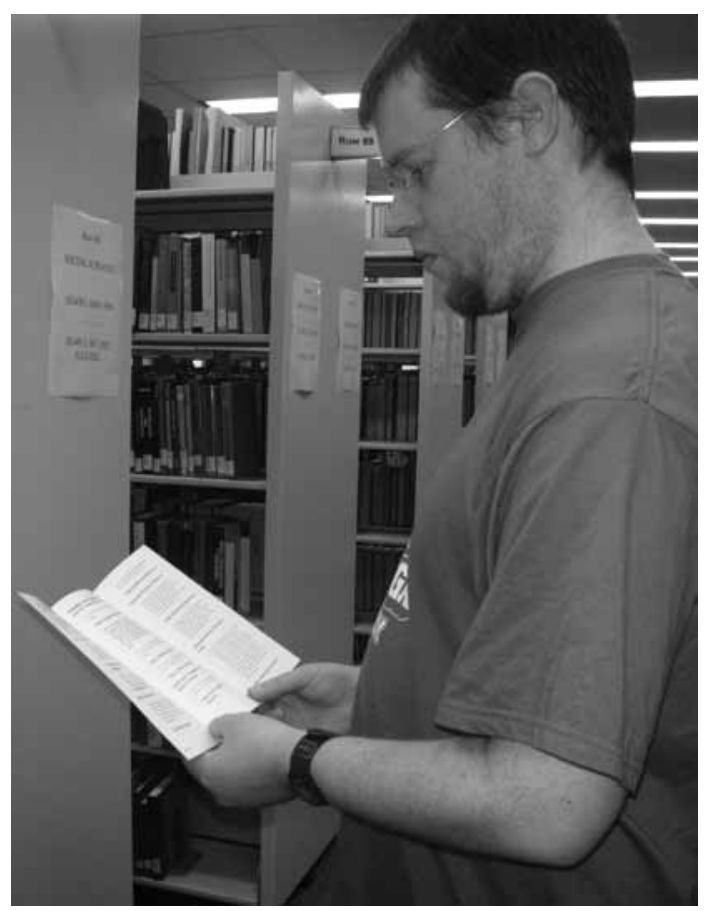

Figure 2. 2D Webpage

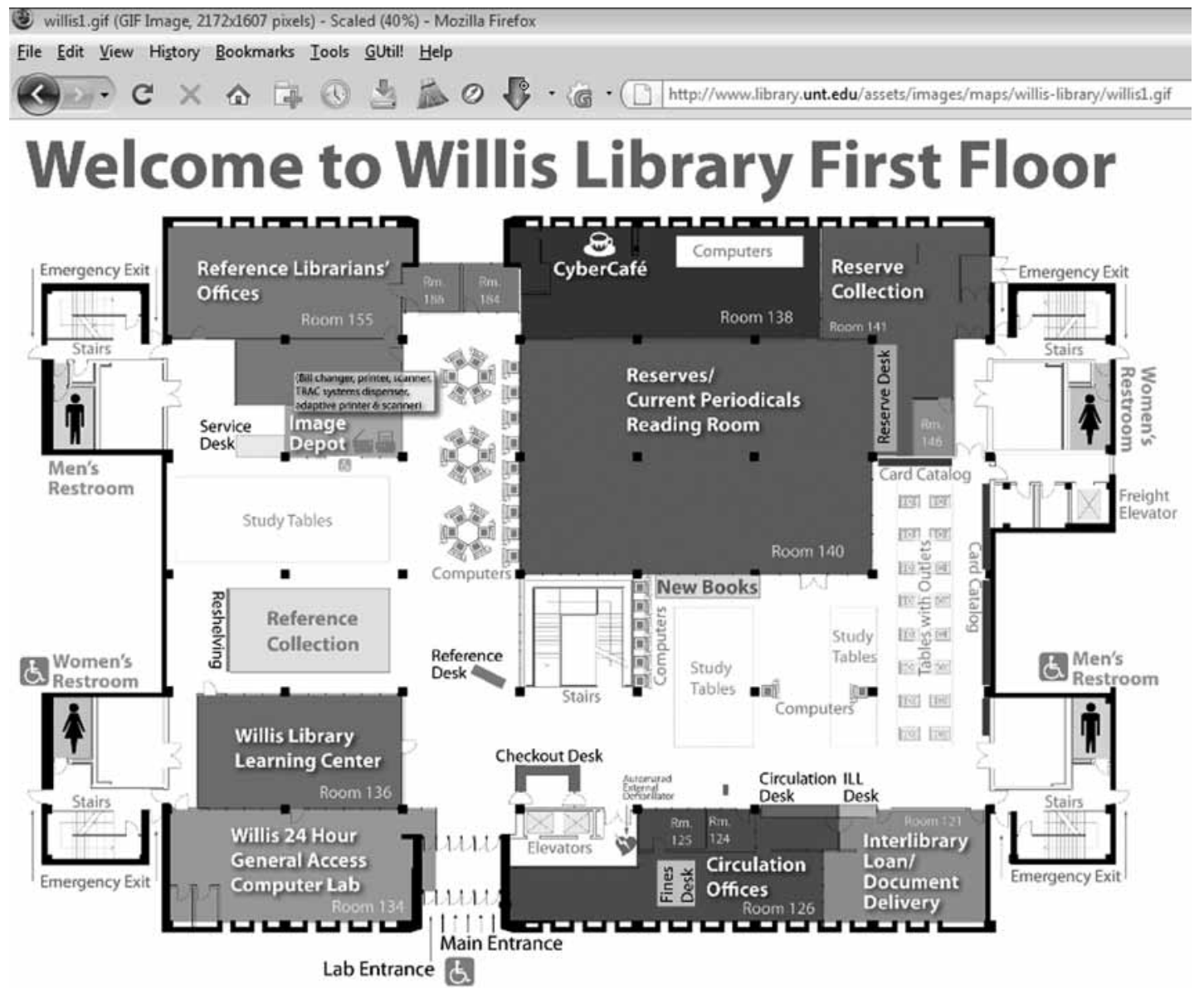


Figure 3. 3D Immersive GUI

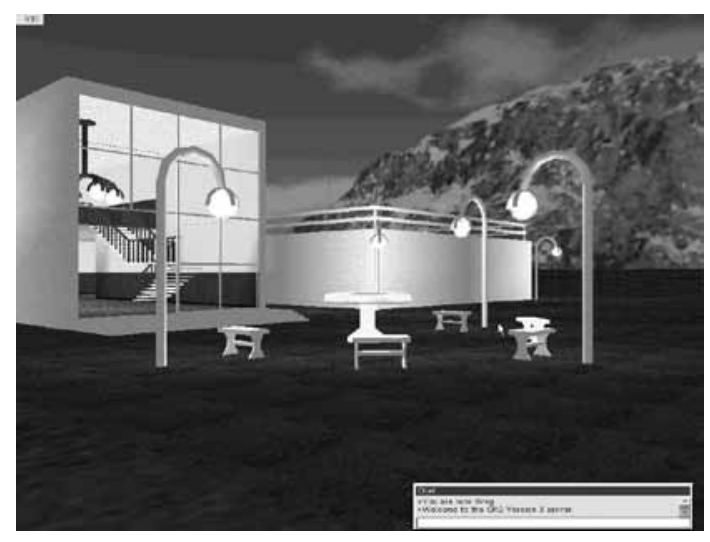

votes for each pair, researchers are able to derive a preference ranking of the objects relative to the given question or scenario. Pairwise comparisons are a common voting method, used in the soft drink taste test, for example. In such a test, the participant is first presented with two cups, one marked A and the other B, with each containing a particular soft drink, the specific brand of which he or she does not know. The administrator informs the participant to take a sip from each of the two cups. Next, the administrator asks the participant, "Which soft drink do you prefer, A or B?" The participant then indicates to the administrator or records in some fashion his or her preference. In short, pairwise comparisons solicit votes of preference by participants. The soft drink taste test example presents participants with only one pairwise comparison, whereas the survey instrument used in this study presented participants with a series or group of comparisons for the respective questions.

The textual content of the survey instrument consists of three questions. Using the same list of scalable objects (the five communication media previously listed), two of the three questions include ten pairwise comparisons each, and the third question solicits descriptive information regarding the participant's age bracket. The survey instrument does not collect data on gender, economic background, or hours spent using particular communication media per week because of a desire to keep the data collection process as brief as possible without sacrificing the overall value of the research. Moreover, research on digital game use indicates that age may be the most important categorical factor in predicting user expectations. ${ }^{31}$ The first question (Q1) asks, "Which type of medium would you rather use for acquiring information on the physical layout of the library?" The second question (Q2) asks,
"Which type of medium would you rather use for receiving instruction on various library services and information literacy skills?" In addition to its textual content, the survey instrument provides graphics exemplifying the scalable objects to guide participants should any confusion on those communication media relative to the given questions arise.

Over a three-week period during the fall 2006 semester, we administered the survey instrument to two participant groups in physical classroom environments on the UNT campus. One participant group had thirty-three participants, the other participant group had nine. The survey process took no more than ten minutes for any participant to complete. Before beginning the survey process, participant groups received brief verbal instructions from the administrator. The instructions focused only on the steps necessary to complete the survey process; no discussion of the communication media occurred beyond pointing to the location of the examples provided in the survey instrument. In addition, if a participant had a question or questions during the survey process, the administrator quietly attended to the individual, independent of the rest of the participant group. In short, administrators approached the survey process as a quasi-formal test situation wherein participants could not pass information to one another in any form. The reason for such an environment is simple-to minimize immediate external peer influence on individual responses to the given stimuli.

\section{DATA ANALYSIS}

Detailed analysis of the data set was conducted using three nonparametric scaling methods: (1) rank-sum scaling of the objects, (2) circular triad analysis to identify inconsistencies, and (3) multidimensional preference mapping to graphically superimpose the affinity of specific participants

Table 1. Participants' Age Brackets

\begin{tabular}{lcc}
\hline Age Bracket & $\begin{array}{c}\text { Participants } \\
(\mathbf{n}=\mathbf{4 2})\end{array}$ & Percentage \\
18 or younger & 1 & $2 \%$ \\
$19-30$ & 18 & $43 \%$ \\
$31-40$ & 13 & $31 \%$ \\
$41-50$ & 5 & $12 \%$ \\
51 or older & 5 & $12 \%$ \\
\hline
\end{tabular}


Table 2. Rank Totals and Scale Scores for Q1

\begin{tabular}{ccc}
\hline Object & Rank Total & Scale Score \\
Min & 42 & 0 \\
1 & 141 & 59 \\
2 & 163 & 72 \\
3 & 157 & 68 \\
4 & 62 & 12 \\
5 & 107 & 39 \\
Max & 210 & 100 \\
\hline
\end{tabular}

with specific objects. ${ }^{32}$ Ultimately, applying these three related yet distinct methods allowed for the desirable triangulation of analytic efforts. Data collected on participants' age brackets were purely descriptive and therefore not addressed in this section; however, those findings are integrated into both the discussion and conclusion sections of this article.

\section{Comparability of Communication Media: Rank-Sum Scaling}

Analysis of Q1 indicated that objects 2 and 3 grouped closely together, with the remaining three spread out across the unidimensional scale (see figure 4). As shown in table 2, rank totals across the forty-two participants' choices for communication media resulted in 62 for object 4, 107 for object 5, 141 for object 1,157 for object 3, and 163 for object 2 . Table 3 shows that rank-sum differences between the five objects range from 6 (the difference between objects 2 and 3) to 101 (the difference between objects 2 and 4). Eight of the ten rank-sum differences are beyond the critical value
Table 3. Rank-Sum Differences for Q1

\begin{tabular}{cccccc}
\hline & $\mathbf{2}$ & $\mathbf{3}$ & $\mathbf{1}$ & $\mathbf{5}$ & $\mathbf{4}$ \\
$\mathbf{2}$ & 0 & & & & \\
$\mathbf{3}$ & 6 & 0 & & & \\
$\mathbf{1}$ & 22 & 16 & 0 & & \\
$\mathbf{5}$ & 56 & 50 & 34 & 0 & \\
$\mathbf{4}$ & 101 & 95 & 79 & 45 & 0 \\
\hline
\end{tabular}

of 16 to reach significance at the $\mathrm{p}<.001$ level. As shown in table 2, rank totals were converted to scale scores on a 0-100 scale and are graphically displayed in figure 4.

Analysis of Q2 indicated that objects 1 and 5 grouped together somewhat closely, while object 2 ranked the highest (see figure 5). Table 4 shows that rank totals across the forty-two participants' responses resulted in 73 for object 4, 122 for object 5, 128 for object 1, 141 for object 3, and 166 for object 2. Table 5 shows that rank-sum differences between the five objects range from 6 (between objects 1 and 5) to 93 (between objects 2 and 4). Eight of the ten rank-sum differences are beyond the critical value of 16 to reach significance at the $\mathrm{p}<.001$. As shown in table 4 , rank totals were converted to scale scores on a 0-100 scale and are graphically displayed in figure 5.

\section{Identifying Inconsistencies: CircularTriads}

The second analytic method focused on identifying any circular triads or intransitive choices indicating inconsistencies between participant responses. By identifying the object or objects that caused several participants to be inconsistent

Figure 4. Unidimensional Display of Scale Scores for Q1

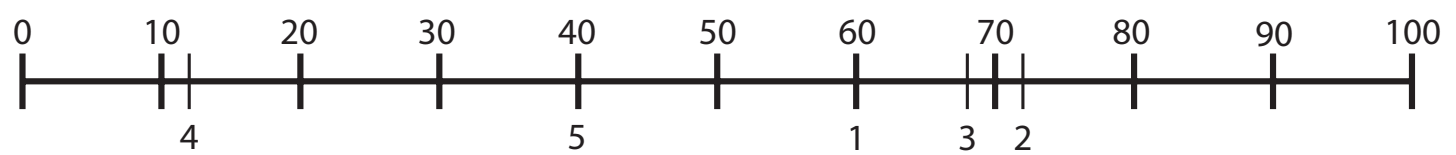

Figure 5. Unidimensional Display of Scale Scores for Q2

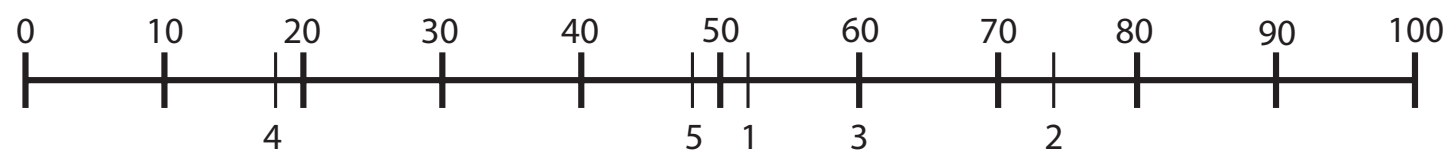


Table 4. Rank Totals and Scale Scores for Q2

\begin{tabular}{|c|c|c|c|}
\hline Object & \multicolumn{2}{|c|}{ Rank Total } & Scale Score \\
\hline Min & \multicolumn{2}{|c|}{42} & 0 \\
\hline 1 & \multicolumn{2}{|c|}{128} & 51 \\
\hline 2 & \multicolumn{2}{|c|}{166} & 74 \\
\hline 3 & \multicolumn{2}{|c|}{141} & 59 \\
\hline 4 & \multicolumn{2}{|c|}{73} & 18 \\
\hline 5 & \multicolumn{2}{|c|}{122} & 48 \\
\hline $\operatorname{Max}$ & \multicolumn{2}{|c|}{210} & 100 \\
\hline \multicolumn{4}{|c|}{ Table 6. Summary of Circular Triad Analysis for Q1 } \\
\hline Object & CT's In & Votes & Scaled \\
\hline 1 & 3 & 99 & 58.93 \\
\hline 2 & 3 & 121 & 72.02 \\
\hline 3 & 4 & 115 & 68.45 \\
\hline 4 & 2 & 20 & 11.90 \\
\hline 5 & 3 & 65 & 38.69 \\
\hline
\end{tabular}

and/or locate a particular participant responsible for a large number of circular triads, we were better able to determine both object scalability and individual participant consistency. For the first two questions, none of the participants or objects were removed for this iteration of analysis; however, future retests may include such actions. Ultimately, this method helped confirm the ranksum scaling results as well as assess the overall quality of the previously untested data collection instrument.

For Q1, forty of the participants were consistent in their choices, whereas two responded with circularity. Table 6 shows a summary of circular triad analysis, including an itemization of objects by the number of circular triads associated with each. Note that the scale values provided mirror those derived from the rank-sum scaling analysis described in the previous section. Object 3 produced the most circular triads (4), whereas object 4 generated the fewest (2).

Table 7 provides a general summary of the circular triad analysis for Q2. Object 2 triggered the most circular triads (7), whereas object 1 initiated the fewest (1). Thirty-six of the participants were consistent in their choices, while six demonstrated circularity.
Table 5. Rank-Sum Differences for Q2

\begin{tabular}{|c|c|c|c|c|c|}
\hline & 2 & 3 & 1 & 5 & 4 \\
\hline 2 & 0 & & & & \\
\hline 3 & 25 & 0 & & & \\
\hline 1 & 38 & 13 & 0 & & \\
\hline 5 & 44 & 19 & 6 & 0 & \\
\hline 4 & 93 & 68 & 55 & 49 & 0 \\
\hline \multicolumn{6}{|c|}{ Table 7. Summary of Circular Triad Analysis for Q2 } \\
\hline Object & & CT's In & Votes & & Scaled \\
\hline 1 & & 1 & 86 & & 51.19 \\
\hline 2 & & 7 & 124 & & 73.81 \\
\hline 3 & & 4 & 99 & & 58.93 \\
\hline 4 & & 5 & 31 & & 18.45 \\
\hline 5 & & 4 & 80 & & 47.62 \\
\hline
\end{tabular}

\section{Scaling Participants and Objects: Multidimensional Preference Mapping}

Multidimensional preference mapping (MDPREF) _ based upon the matrix theorem of Eckhart and Young and also known as geometric factor analysis-was applied to the data set to situate the objects and the participants in the same analytic, psychological space. ${ }^{33}$ The primary motivation for using this method was to provide a visualization of specific subgroups of participants with specific objects. The various distances between subgroups and objects represent participants' perceptions of similarity and dissimilarity between points. As the remainder of this section details, MDPREF analysis indicated that, for both questions, each of the five objects maintained different levels of distinctness among one another and participant subgroups.

MDPREF analysis of Q1 (see figure 6) indicated various alignments. For example, objects 1 and 5 collocate on the left side of the y-axis, with objects 2, 3, and 4 collocating on the right side of the y-axis. This specifies some level of significant difference between the former objects and the latter. In addition, because each of the three latter objects is located within the right-side plots, some level of alignment is also present among these objects. The most interesting observation is that object 3 , as opposed to object 2 , lies within the lower-right-hand plot of the graph; this space also includes the majority of participants. 
Figure 6. Plot of Objects and Participants in First Two Dimensions for Q1

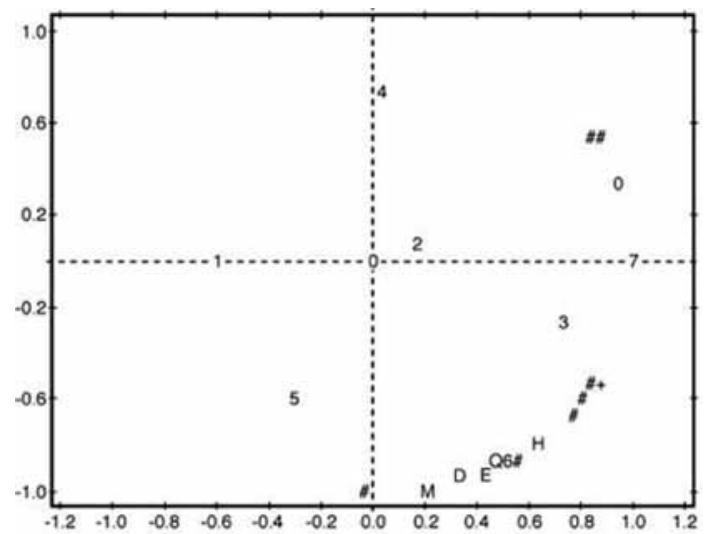

Note: Multiple points identified as \#. First five points are objects, and remaining points are participants.

Figure 7 shows that, for Q2, objects 1 and 5 are collocated within the upper-left quadrant of the plot, objects 4 and 2 are closer to one another within the lower-left quadrant of the plot, and object 3 is located to the right side of the $\mathrm{x}$-axis. In addition, the participants' locations are more widespread than Q1; however, they do loosely mirror Q1 results, indicating an affinity for particular communication media across both questions. Furthermore, it is noteworthy that the participants appear to align closest with object 3, as they did with Q1.

\section{DISCUSSION}

This section presents select interpretations related to the findings in the data analysis section. Overall, the three interpretations deemed most noteworthy are that (1) the 2D webpage was the preferred communication medium across both questions; (2) preference for the audio-only communication medium was remarkably low across both questions; and (3) the 3D immersive GUI received considerable preference for use in relation to Q1, as well as adequate evidence warranting further research on its use in Q2 instruction.

\section{Q1: Library Layout}

Both rank-sum analysis and circular-triad analysis established that the majority of participants preferred the existing 2D webpage communication medium, with the 3D immersive GUI following close behind. The authors initially expected a portable, paper-based document, such as a pamphlet, to be preferred, since the question focuses
Figure 7. Plot of Objects and Participants in First Two Dimensions for Q2

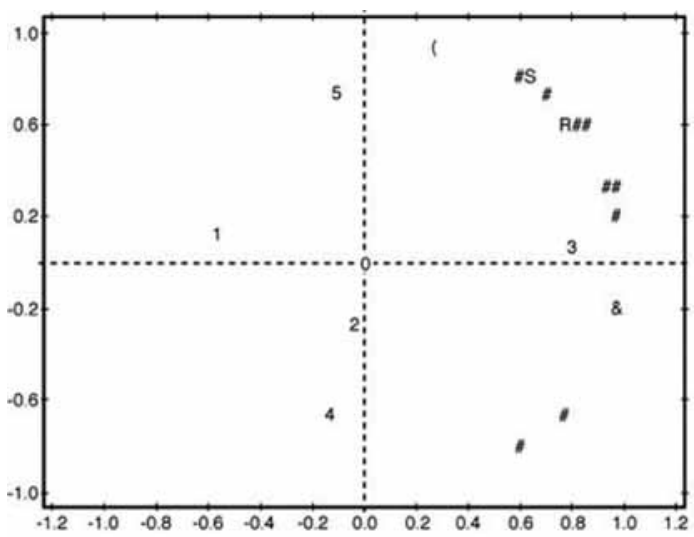

Note: Multiple points identified as \#. First five points are objects, and remaining points are participants.

on concepts related to spatial information. Therefore it is noteworthy that both the $2 \mathrm{D}$ webpage and 3D immersive GUI received higher preference rankings. Also, considering the increase in discourse throughout the LIS community championing podcasting, the low preference ranking of the audio-only communication medium is interesting. In addition, MDPREF results indicate that participants' placements appear most aligned, or generally closest, with the 3D immersive GUI. Such placement suggests a level of preference for this communication medium not evident in the rank-sum and circular-triad results.

\section{Q2: Information Literacy}

In both rank-sum and circular-triad analyses, the 2D webpage ranked significantly higher than the other four communication media. This outcome was particularly interesting, since this question concerns users involved in tasks associated with learning information literacy skills. It ran contrary to the researchers' initial expectation that Millennial users, the largest subset of the sample, would be more likely to choose the 3D immersive GUI over other communication media. Also, like Q1, a significant lack of preference toward audio-only is noticeable. Perhaps users over eighteen years old (all but one of the study participants) do not associate this communication medium with educational tasks. In addition, 2D webpage, 3D immersive GUI, and A/V each involved similar numbers of circular triads; however, as previously stated in the data analysis section, the decision was made not to remove them from this iteration of analysis. Nonetheless, this observation suggests that participants 
had a difficult time making firm decisions on the applicability of each of these communication media relative to one another. MDPREF analysis did not further reveal the relationship between the 2D webpage, 3D immersive GUI, and A/V—although, like Q1, it indicated a certain level of enthusiasm for the 3D immersive GUI not initially evident in the rank-sum and circular-triad analyses.

\section{CONCLUSION}

This discussion addressed research on academic library user preferences related to five communication media used to facilitate two forms of library instruction. The corresponding study began with a survey instrument administered to forty-two participants over a three-week period in the fall of 2006. Upon completion of the data collection process, three separate scaling methods were applied to the data set. Analysis indicates an overall preference for the 2D webpage approach, as well as notable enthusiasm for the 3D immersive GUI, the principal user interface throughout current digital gaming technologies. Analysis also shows an overall lack of preference toward the audio-only communication medium.

It is noteworthy that the scaling methods used here allow one to quantify user preferences toward an object, concept, and so on. For example, Participant A likes the 2D webpage more than audio-only and paper-based media. In formal statistics, such data are of the ordinal type. Ordinal data require nonparametric analysis; however, parametric methods are traditionally preferred in LIS research. ${ }^{34}$ Parametric methods allow one to generalize findings to a larger population, whereas nonparametric methods are more or less free from assumptions and therefore do not allow for generalizations. Nonetheless, scaling methods offer LIS researchers a variety of important perspectives when properly applied. For example, researchers debating whether or not to conduct extensive statistical studies in areas similar to those presented here could adopt these or related methods for an initial small-scale test period that would allow them to gauge preliminarily the accuracy of their incoming assertions. In such cases, scaling methods would facilitate a sort of statistical litmus test. Thus, while not as powerful or robust as parametric methods, given an adequate participant to objects ratio, the results of these nonparametric methods can guide researchers and other decision makers in determining whether or not more advanced, resource-intensive, statistical studies are appropriate.

Ultimately, in this study it is most important that the $2 \mathrm{D}$ webpage received the highest prefer- ence ranking across both questions. Furthermore, the significant lack of interest in audio-only is a bit startling, considering the enthusiasm by many LIS practitioners for podcasting in an educational capacity. Perhaps practitioners should investigate more extensively the application of this communication medium for such purposes. In addition, this study indicates noteworthy preference for application of a 3D immersive GUI in library instruction, particularly any educational efforts involving the communication of spatial information concerning a physical library facility. Also, considering the results of all three analytic methods for Q2, the same likelihood may also exist for the 3D immersive GUI as a medium for information literacy instruction. In the future, the authors plan to continue research in the areas of digital gaming and library instruction, in particular improving the data collection instrument through both content expansion and migration onto a digital platform; collecting larger data sets for analysis with similar methods; and interviewing instructional librarians, library administrators, and digital game designers to solicit their perspectives on the findings in this article as well as future outcomes of research.

\section{References and Notes}

1. Lauren Barack, "Gaming at Your Library," School Library Journal 51, no. 7 (July 2005): 22, www.schoollibrary journal.com/article/CA621772.html (accessed Dec. 1, 2006); Christy Branston, "From Game Studies to Bibliographic Gaming: Libraries Tap into the Video Game Culture," Bulletin of the American Society for Information Science and Technology 32, no. 4 (Apr./May 2006): 24-26, 29; Ameet Doshi, "How Gaming Could Improve Information Literacy," Computers in Libraries 26, no. 5 (2006): 14-17, www.infotoday.com/cilmag/ may06/Doshi.shtml (accessed Dec. 1, 2006); Donald T. Hawkins and Barbara Brynko, "Gaming: The Next Hot Technology for Libraries?” Information Today 23, no. 6 (June 2006): 1-51; Andrew Hinton, "We Live Here: Games, Third Places and the Information Architecture of the Future," Bulletin of the American Society for Information Science and Technology 32, no. 6 (Aug./ Sept. 2006): 17-21; Metropolitan Library System, "Gaming, Learning, and Libraries: 2005 Symposium," Metropolitan Library System, www.gaminginlibraries. org/2005symposium (accessed Nov. 11, 2006); Lauree Padgett, "Gaming for Info Literacy," Information Today 23, no. 5 (May 2006): 46-47; Michael Stephens, "Promoting Gaming Programs in Libraries," Marketing Library Services 20, no. 2 (Mar./Apr. 2006), www .infotoday.com/MLS/mar06/Stephens.shtml (accessed Nov. 8, 2006); Lynn Sutton and H. David Womack, "Got Game? Hosting Game Night in an Academic Library," College \& Research Libraries News 67, no. 3 (2006): 173-76; Ray Uzwyshyn, "Networked 3D Game Possibilities," http://informationvisualization. typepad.com (accessed July 13, 2006).

2. Howard Dillon, "Organizing the Academic Library for Instruction," Journal of Academic Librarianship 1, 
no. 4 (1975): 4-7; Michael Lorenzen, "Brief History of Library Instruction in the United States," Illinois Libraries 83, no. 2 (2001): 8-18.

3. James G. Jones and Stephen C. Bronack, "Rethinking Cognition, Representations, and Processes in 3D Online Social Learning Environments," Games and Simulations in Online Learning, ed. David Gibson, Clark Aldrich, and Marc Prensky (Hershey, Pa.: Idea Group, 2006); David Williamson et al., "Video Games and the Future of Learning," www.academiccolab .org/resources/gappspaperl.pdf (accessed Dec. 1, 2006); Constance Steinkuehler, "Cognition and Learning in Massively Multiplayer Online Games: A Critical Approach," http://website.education.wisc.edu/ steinkuehler/thesis.html (accessed Dec. 1, 2006).

4. Ogechi Nnadi, Ute Fischer, Michael Boyce, and Michael Nitsche, "Effect of Dynamic Camera Control on Spatial Reasoning in 3D Spaces," Proceedings of the 2008 ACM SIGGRAPH Symposium on Video Games (Los Angeles: ACM, 2008); Pippin Barr et al., "Playing the Interface: A Case Study of Grand Theft Auto: San Andreas," Proceedings of the 20th Conference of the Computer-human Interaction Special Interest Group (CHISIG) of Australia on Computer-human Interaction: Design: Activities, Artefacts and Environments (Sydney, Australia: ACM, 2006); Jo Bryce and Jason Rutter, "Spectacle of the Deathmatch: Character and Narrative in First-Person Shooters," Screenplay: Cinemal Videogames/Interfaces, ed. Geoff King and Tanya Krzywinska (New York: Wallflower, 2002).

5. Nora S. Newcombe and Janellen Huttenlocher, Making Space: The Development of Spatial Representation and Reasoning (Cambridge: MIT Press, 2000); Leonidas Deligiannidis and Robert J. K. Jacob, "An Immersive Environment for the Vase Museum" (paper presented at the HCI '05 International Conference on Human-Computer Interaction: 2005 World Congress in Applied Computing, Las Vegas, Nev., June 20-23 2005); Leonidas Deligiannidis and Robert J. K. Jacob, "The London Walkthrough in an Immersive Digital Library Environment" (paper presented at the 2005 International Conference on Modeling, Simulation, and Visualization Methods (MSV '05), Las Vegas, Nev., June 27-30 2005); Paolo Paolini et al., "Visiting a Museum Together: How to Share a Visit to a Virtual World," Journal of the American Society for Information Science 51, no. 1 (2000): 33-38.

6. James G. Greeno, "On Claims That Answer the Wrong Questions," Educational Researcher 26, no. 1 (1997): 5-17; Constance Steinkuehler, "Cognition as (Inter)Action in the Social \& Material World," http:// website.education.wisc.edu/steinkuehler/cogtheory. html (accessed Dec. 1, 2006); Elisabeth Davies, "Communities of Practice," Theories of Information Behavior, ed. Karen E. Fisher, Sanda Erdelez and Lynne McKechnie (Medford, N.J.: Information Today, 2005); Sharon J. Derry and Constance A. Steinkuehler, "Cognitive and Situative Theories of Learning and Instruction," Encyclopedia of Cognitive Science, ed. L. Nadel (London: Nature Publishing Group, 2003); James Paul Gee, An Introduction to Discourse Analysis: Theory and Method (London/New York: Routledge, 1999); John Dewey, How We Think (Lexington, Mass.: Heath, 1933); George Kelly, A Theory of Personality: The Psychology of Personal Constructs (New York: Norton, 1963); Jerome S. Bruner, "The Act of Discovery," Harvard Educational Review 31 (1961): 21-32; Lev S.
Vygotsky, Mind in Society: The Development of Higher Psychological Processes (Cambridge: Harvard Univ. Pr., 1978).

7. Rayne A. Sperling et al., "Animations as Learning Tools in Authentic Science Materials," International Journal of Instructional Media 30, no. 2 (2003): 21321.

8. Jerry Johnson, "Information Workplace of the Future," Pacific Northwest National Laboratory, www.pnl.gov/ ima/IMA-2004-12-07.ppt (accessed Feb. 1, 2006); M. J. Koepp et al., "Evidence for Striatal Dopamine Release during a Video Game," Nature 393, no. 6682 (1998): 266-68; Merrilea J. Mayo, "Games for Science and Engineering Education," Communications of the ACM 50, no. 7 (2007): 31-35.

9. Gary Marchionini and Hermann Maurer, "The Role of Digital Libraries in Teaching and Learning," Communications of the ACM 38, no. 4 (1995): 67-75.

10. Jane Keefer, "The Hungry Rats Syndrome: Library Anxiety, Information Literacy and the Academic Reference Process," RQ 32, no. 3 (Spring 1993): 333-39.

11. Tammy J. Eschedor Voelker, "The Library and My Learning Community: First Year Students' Impressions of Library Services," Reference \& User Services Quarterly 46, no. 2 (2006): 72-80.

12. James R. Bettman and Pradeep Kakkar, "Effects of Information Presentation Format on Consumer Information Acquisition Strategies," Journal of Consumer Research 3, no. 4 (1977): 233-40; Gabriel Biehal and Dipankar Chakravarti, "Information-Presentation Format and Learning Goals as Determinants of Consumers' Memory Retrieval and Choice Processes," The Journal of Consumer Research 8, no. 4 (1982): 431-41.

13. James G. Jones and Stephen C. Bronack, "Rethinking Cognition, Representations, and Processes in 3D Online Social Learning Environments."

14. Voelker, "The Library and My Learning Community."

15. Barbara Dewey, Library User Education: Powerful Learning, Powerful Partnerships (Lanham, Md.: Scarecrow, 2001); Michael B. Eisenberg and Robert E. Berkowitz, Information Problem Solving: The Big Six Skills Approach to Library and Information Skills Instruction (Norwood, N.J.: Ablex, 1990)

16. Arie C. Koelewyn and Katherine Corby, "Citation: A Library Instruction Computer Game,” RQ 22, no. 2 (1982): 171-74.

17. Pearson Education provides games of this type at www.funbrain.com (accessed Dec. 1, 2006). See also Dyann Schmidel and Wanda Wojcik, "High School Ace: The Academic Homepage for High School Students," http://highschoolace.com/ace/ace.cfm (accessed Dec. 1, 2006); The Cybrarian, "Cybrarian Kids' Educational Curriculum Site," www.cybrary.org (accessed Apr. 4, 2007); Pierre Cubaud, Claire Thiria, and Alexandre Topol, "Experimenting a 3D Interface for the Access to a Digital Library," Third ACM Conference on Digital Libraries (Pittsburgh, Pa.: ACM, 1998); Infoisland.org, "Infoisland.Org: Second Life Library 2.0," http://infoisland.org (accessed Dec. 1, 2006); Mark D. Puterbaugh, "The Virtual Bibliographic Instruction Project," Eastern University, www.eastern .edu/library/www/services/chat/vbiproject.shtml (accessed Dec. 1, 2006); Yumetech Inc., "DiRBS: The Digital Rare Book Library System," www.yumetech .com/projects/dirbs.html (accessed Dec. 1, 2006); J. 
Erdman, "Reference in a 3-D Virtual World: Preliminary Observations on Library Outreach In 'Second Life" The Reference Librarian 47, no. 2 (2007): 29-39; K. Swanson, "Second Life: A Science Library Presence in Virtual Reality," Science \& Technology Libraries 27, no. 3 (2007): 79-86.

18. Andrew Lewis, "Marketing Library Computers to Young Children Using Multimedia," New Review of Children's Literature and Librarianship 11, no. 1 (2005): 47-62.

19. Voelker, "The Library and My Learning Community"; Doshi, "How Gaming Could Improve Information Literacy."

20. Stephen Abram and Judy Luther, "Born with the Chip," Library Journal 129, No. 8 (May, 2004): 34-37, www.libraryjournal.com/article/CA411572 .html (accessed Oct. 29, 2007).

21. Steve Jones, "Let the Games Begin: Gaming Technology and Entertainment among College Students," (Washington, D.C.: Pew Internet and American Life Project, 2003), www.pewinternet.org/pdfs/PIP_College_Gaming_Reporta.pdf (accessed Nov. 18, 2007).

22. Diana Oblinger and James L. Oblinger, "Educating the Net Generation," (EDUCAUSE, 2005), http://bibpurl .oclc.org/web/9463 (accessed July 20, 2007).

23. N. Rise, "The Interactive Children's Library of the Future," Bibliotekspressen 10 (2006): 16-17; Chris Dede, "Planning for Neomillennial Learning Styles," EDUCAUSE Quarterly 28, no. 1 (2005), www.educause .edu/apps/eq/eqm05/eqm0511.asp?bhcp=1 (accessed Oct. 29, 2007).

24. Robert J. Sternberg, Cognitive Psychology, 4th ed. (Belmont, Calif:: Thomson Wadsworth, 2006).

25. Rhonda Christensen and Gerald Knezek, "Stages of Adoption for Technology in Education," Computers in New Zealand 11, no. 3 (1999): 25-29; Fred D. Davis, Richard P. Bagozzi, and Paul R. Warshaw, "User Acceptance of Computer Technology: A Comparison of Two Theoretical Models," Management Science 35, no. 8 (1989): 982-1003; Kieran Mathieson, "Predicting User Intentions: Comparing the Technology Acceptance Model with the Theory of Planned Behavior," Information Systems Research 2, no. 3 (1991): 173-91; Viswanath Venkatesh et al., "User Acceptance of
Information Technology: Toward a Unified View," MIS Quarterly 27, no. 3 (2003): 425-78; Michael Leicht and Vicki Sauter, "Managing User Expectations," www.umsl.edu/ sauterv/analysis/user_expectations .html (accessed Nov. 1, 2007).

26. Stanley Smith Stevens, Psychophysics: Introduction to Its Perceptual, Neural, and Social Prospects (New York: Wiley, 1975).

27. Amos Tversky, "Features of Similarity," Psychological Review 84, no. 4 (1977): 327-52; Stanley Smith Stevens, "The Direct Estimation of Sensory Magnitudes-Loudness," American Journal of Psychology 69, no. 1 (1956): 1-25.

28. Michael B. Eisenberg, "Magnitude Estimation and the Measurement of Relevance" (PhD dissertation, Syracuse University, 1986); Guillermo A. Oyarce, "A Study of Graphically Chosen Features for Representation of TREC Topic-Document Sets" (PhD dissertation, University of North Texas, 2000); Abebe Rorissa, "Perceived Features and Similarity of Images: An Investigation into Their Relationships and a Test of Tversky's Contrast Model" (PhD dissertation, University of North Texas, 2005); Mark E. Rorvig, "An Experiment in Human Preferences for Documents in a Simulated Information System (Choice, Simple Scalability)" (PhD dissertation, University of California, Berkeley, 1985).

29. University of North Texas-Institutional Research and Accreditation, "Enrollment Fact SheetFall 2006," www.unt.edu/ir_acc/Enrollment\%20 Fact\%20Sheet/2006-Fall_Enrollment-Fact_Sheet .html\#spring5 (accessed Aug. 3, 2007).

30. Peter Dunn-Rankin et al., Scaling Methods, 2nd ed. (Mahwah, N.J.: Lawrence Erlbaum, 2004).

31. Entertainment Software Association, "Game Player Data," www.theesa.com/facts/gamer_data.php (accessed Oct. 29, 2007).

32. Dunn-Rankin et al., Scaling Methods.

33. Carl Eckhart and Gale Young, "The Approximation of One Matrix by Another of Lower Rank," Psychometrika 1, no. 3 (1936): 211-18.

34. Liwen Vaughan, Statistical Methods for the Information Professional (Medford, N.J.: Information Today, 2005).

\section{STALKING THE WILD APPEAL FACTOR CONTINUED FROM PAGE 246}

(accessed Nov. 8, 2008).

7. LibraryThing, "Shelfari Spam," post on Thingology, Nov. 8, 2007, www.librarything.com/thingology/2007/11/shelfari-spam-basically-social.php (accessed Nov. 8, 2008).

8. John Cook, "Amazon.com buys Shelfari, a startup for book lovers," http://seattlepi.nwsource.com/business/376443_amazonshelfari26.html (accessed Nov. 7, 2008).

9. GoodReads, "Press Information," www.goodreads. com/about/press (accessed Nov. 7, 2008).

10. Leah Dodd, personal communication with the author, Apr. 23, 2008.

11. Alicia Ahlvers, personal communication with the author, Nov. 7, 2008.

12. Bryan Jones, personal communication with the author, Apr. 23, 2008.
13. Lesa Holstine, personal communication with the author, Apr. 23, 2008.

14. Robin Beerbower, personal communication with the author, Apr. 23, 2008.

15. Susan Smith, personal communication with the author, Apr. 28, 2008.

16. Neil Hollands, "Shelfari or LibraryThing for Book Groups," post on Book Group Buzz, June 13, 2008, http://bookgroupbuzz.booklistonline. com/2008/06/13/shelfari-or-library-thing-for-bookgroups (accessed Nov. 6, 2008).

17. Sharon L. Cosentino, "Folksonomies: Path to a Better Way?" Public Libraries 47, no. 2 (Mar./Apr. 2008): 44.

18. Michael Stephens, "Taming Technolust: Ten Steps for Planning in a 2.0 World," Reference \& User Services Quarterly 47, no. 4 (Summer 2008): 314. 